05

\title{
Рефракция на неоднородности газа при лазерном нагреве металла
}

\author{
(C) V. Trofimov ${ }^{1}$, H.Н. Розанов ${ }^{2}$, Y. Yang ${ }^{1}$, C.В. Федоров ${ }^{2}$, J. Yu $^{1}$, Н.A. Веретенов ${ }^{1}$ \\ ${ }^{1}$ South China University of Technology, \\ 510640 Guangzhou China Guangzhou, China \\ ${ }^{2}$ ФТИ им. А.Ф. Иоффре РАН, \\ 194021 Санкт-Петербург, Россия \\ e-mail: nnrosanov@mail.ru
}

Поступила в редакцию 27.12.2021 г.

В окончательной редакции 27.12.2021 г.

Принята к публикации 10.01.2022 г.

\begin{abstract}
Проведен анализ распределения показателя преломления вблизи границы нагреваемого лазерным излучением металла. Найдены градиент показателя преломления газа, вызванный потоком тепла, и распределение показателя преломления в неоднородно нагреваемом газе. Выполнена оценка сноса лазерного пучка вследствие рефракции излучения в газе, показывающая возрастание величины сноса для узких лазерных пучков.
\end{abstract}

Ключевые слова: рефракция в газе, нагрев газа, лазерный нагрев металла.

DOI: $10.21883 /$ OS.2022.04.52280.55-21

В настоящее время интенсивно развиваются методы 3D-печати, основанные на воздействии лазерного излучения на металлы, пластмассы и другие вещества. Процессы нагрева вещества (в дальнейшем рассматриваем металл) во многом определяют качество изготавливаемых образцов. При теоретическом анализе процесса нагрева, как правило, полагают, что оптическое излучение в плоскости образца имеет заданный (гауссов) профиль и на него не оказывают влияние процессы распределения температуры в газе, охлаждающем поверхность металла [1-7]. Определенным оправданием служит крайняя малость коэффициента поглощения газа и близость его показателя преломления к единице. Однако достигаемая в прецизионных устройствах точность печати достигает величин микронного диапазона [8], что требует учета влияния наличия газа в установке (камере) на распространение излучения. Этой задаче и посвящена настоящая статья.

Получение оценок эффекта облегчается слабостью неоднородности газа. Это позволяет, прежде всего, оценить саму вызванную лазерным нагревом неоднородность газа, который мы будем считать не ионизированным. При этом допустимым оказывается полное пренебрежение поглощением излучения в самом газе, так что его нагрев обусловлен потоком тепла от поверхности металла. Центральную часть широкого лазерного пучка можно моделировать плоской волной. Тогда стационарный нагрев газа описывается уравнением теплопроводности

$$
\frac{d}{d z}\left[\Lambda \frac{d T}{d z}\right]+w_{g}=0, \quad w_{g}=u \rho c_{v}\left(T_{g}-T\right), \quad z<0
$$

Координата $z$ направлена ортогонально к поверхности металла, расположенной при $z=0$. Член $w_{g}$ описывает скорость охлаждения газа, т.е. скорость замены нагретого газа холодным с температурой $T_{g}$ (уменьшение количества тепла в газе). Он пропорционален разности температур холодного и нагретого газа $T_{g}-T$, его плотности $\rho$, теплоемкости при постоянном объеме $c_{v}$. Параметр $u$ с размерностью $\mathrm{s}^{-1}$ пропорционален скорости охлаждающего газа $v$; для образца размера $w$ получим $u=v / w$.

В пренебрежении температурной зависимостью теплофизических параметров решение уравнения (1) записывается в виде

$$
T=T_{g}+A \exp (\gamma z), \quad T(z=-\infty)=T_{g}, \quad \gamma^{2}=u \rho c_{v} / \Lambda .
$$

Для учета плавной (медленной) температурной зависимости этих параметров в полуклассическом приближении (ВКБ) можно сделать замену

$$
\exp (\gamma z) \rightarrow \exp \left(\int^{z} \gamma(T(z)) d z\right)
$$

Мы не будем использовать это приближение, так как для оценок форма (2) оказывается достаточной.

Постоянная $A$ в (2) определяется, вообе говоря, сшиванием на границе газа с металлом, так что следовало бы решать совместно уравнения теплопроводности для газа и металла. Однако для оценок будем считать, что при $z=0$ температура газа равна граничной температуре металла $T_{m}$. Последняя вычисляется отдельно, но она известна и из экспериментов, так что будем считать ее заданной.

Тем самым мы получили оценку для температурного профиля в газе. Теперь можно оценить профиль показателя преломления охлаждающего газа. Для $\mathrm{Ar}$ при нормальных условиях температурная зависимость 
показателя преломления $n$ и диэлектрической проницаемости $\varepsilon$ следующая [9]:

$$
\begin{gathered}
n(T)=1.000284-\alpha_{n}\left(T-T_{0}\right), \quad \alpha_{n}=10^{-6}\left(K^{-1}\right), \\
\varepsilon(T)=n^{2}(T) \approx 1-2 \alpha_{n}\left[\left(T_{g}-T_{0}\right)+\left(T_{m}-T_{g}\right) \exp (\gamma z)\right] .
\end{gathered}
$$

Эти соотношения достаточны для оценки сдвига (рефракции) лазерного излучения при его наклонном падении и прохождении слоя газа с неоднородным показателем преломления; оценки показывают, что другой фактор - изменение коэффициента отражения - в условиях охлаждения металла аргоном практически не проявляется.

В приближении геометрической оптики из закона преломления Снеллиуса в плоскослоистой среде с диэлектрической проницаемостью $\varepsilon(z)$ поперечная координата луча в общем случае описывается [10] выражением

$$
x(z)=x_{0}+\sin \theta_{0} \int_{z_{0}}^{z} \frac{d z}{\sqrt{\varepsilon(z) / \varepsilon_{0}-\sin ^{2} \theta_{0}}} .
$$

Здесь $\theta_{0}-$ угол падения на вход неоднородного слоя $z=z_{0}$ и $\varepsilon_{0}=\varepsilon(0)$ (при нормальном падении $\theta_{0}=0$ ). В нашем случае

$$
\varepsilon(z) \approx a+b \exp (\gamma z)
$$

где

$$
\begin{gathered}
a=1-2 a_{n}\left(T_{g}-T_{0}\right), \quad b=-2 a_{n}\left(T_{m}-T_{g}\right), \\
b^{2} \ll a^{2} \sim 1 .
\end{gathered}
$$

Тогда

$$
\begin{aligned}
x(z) \approx & x_{0}+\sin \theta_{0}\left\{A\left(z-z_{0}\right)\right. \\
& \left.+(B / \gamma)\left[\exp \left(\gamma\left(z-z_{0}\right)\right)-1\right]\right\} .
\end{aligned}
$$

Сдвигом луча вследствие рефракции можно считать величину, пропорциональную только коэффициенту нагрева металла, $B=-b / 2=a_{n}\left(T_{m}-T_{g}\right)$ :

$$
\delta x=\sin \theta_{0}(B / \gamma)[\exp (\gamma L)-1] \approx B L\left(1+\frac{1}{2} \gamma L\right) \sin \theta_{0} .
$$

Последнее выражение в (9) справедливо при достаточно малой толщине слоя неоднородного газа $L(\gamma L \ll 1)$.

Приведем численные оценки для характерных параметров: $\Lambda=17.72 \times 10^{-3} \mathrm{~W} /(\mathrm{m} \cdot \mathrm{K}), \quad \rho=1.78 \mathrm{~kg} / \mathrm{m}^{3}$, $c_{v}=17.7 \times 10^{-3} \mathrm{~W} \cdot \mathrm{s}(\mathrm{kg} \cdot \mathrm{K})[11], v=10 \mathrm{~cm} / \mathrm{s}$, разности температур $T_{m}-T_{g}=1200 \mathrm{~K}$. Примем толщину слоя охлаждающего газа $L=2 \mathrm{~cm}$ и ширину пучка $w=4 \mathrm{~cm}$. В этих условиях вызванный градиентом температуры снос луча для широкого пучка $\delta x \approx 24 \cdot \sin \theta_{0} \mu \mathrm{m}$. Это сопоставимо с указанной выше точностью прецизионной печати, что подчеркивает важную роль учета неоднородности газа для повышения этой точности. Для более узких пучков, например, с шириной $w=20 \mu \mathrm{m}$, численное значение сноса столь велико, что соотношение (9) формально приводит к величине сноса, превышающей ширину самого пучка. Этот вариант выходит за рамки принятого плосковолнового приближения и тем самым этот случай требует отдельного анализа. Но он, безусловно, свидетельствует о существенности и обязательности учета рефракции тонких пучков лазерного излучения вблизи поверхности нагреваемого металла.

\section{Финансирование работы}

Исследование поддержано

грантом РФФИ-21-52-53009 ГФЕН.

\section{Конфликт интересов}

Авторы заявляют, что у них нет конфликта интересов.

\section{Список литературы}

[1] А.А. Углов, И.Ю. Смуров, А.М. Лашин, А.Г. Гуськов. Моделирование теплофизических процессов импульсного лазерного воздействия на металлы (Наука, М., 1991), c. $1-288$.

[2] A.M. Prokhorov, V.I. Konov, I. Ursu, I.N. Mihailescu. Laser Heating of Metals (CRC Press, Taylor \& Francis Group, Boca Raton, FL, 1990), p. 1-135.

[3] G.G. Gladush, I. Smurov. Physics of Laser Material Processing. Theory and Experiment (Springer-Verlag, Berlin Heidelberg, 2011), p. 1-526.

[4] W.M. Steen, J. Mazumder. Laser Material Processing. 4th ed. (Springer, London, 2010), p. 1-342.

[5] B.S. Yilbas, A.Y. Al-Dweik, N. Al-Aqeeli, H.M. Al-Qahtani. Laser Pulse Heating of Surfaces and Thermal Stress Analysis (Springer, Heidelberg, 2014), p. 1-176.

[6] M. Gouge, P. Michaleris. Thermo-Mechanical Modelling of Additive Manufacturing (Elsevier Inc., Cambridge, MA, 2018), p. $1-50$.

[7] J. M. Dowden. The Mathematics of Thermal Modeling. An Introduction to the Theory of Laser Material Processing (Chapman \& Hall/CRC, Boca Raton, FL, 2001), p. 1-155.

[8] Seok Kim, Do Hyeog Kim, Wonpyo Kim, Young Tae Cho, Nicholas X. Fang. Int. J. Precision Engineering and Manufacturing-Green Technol., 8, 303-326 (2021) (https://bmf3d.com/high-precision-micro-3d-printers/).

[9] T. Larsén. Z. Physik., 88, 389-394 (1934).

[10] М. Борн, Э. Вольф. Основы оптики (Наука, М., 1973).

[11] https://gsssd-rosatom.mephi.ru/DB-tp-02/Ar.php 